\title{
Instability of a plane conducting free surface submitted to an alternating magnetic field
}

\author{
By Y. FAUTRELLE AND A. D. SNEY D \\ ${ }^{1}$ Ecole Nationale Supérieure d'Hydraulique et de Mécanique de Grenoble, BP 95, \\ 38402 St Martin d'Hères, Cedex France \\ ${ }^{2}$ University of Waikato, Private Bag 3105, Hamilton, New Zealand
}

(Received 1 September 1997 and in revised form 13 March 1998)

This paper considers the stability of a horizontal liquid-metal free surface in the presence of a horizontal alternating magnetic field. A weak formulation is used to derive a generalized Mathieu-Hill equation for the evolution of surface perturbations. Previous studies which rely on time-averaging the electromagnetic force over one field cycle have predicted a generally weak instability, but we find much larger growth rates near the resonances, where the surface wave frequency is an integral multiple of the field frequency. The method can be extended to include viscous and ohmic damping; the former has little effect, while the latter damps all waves except those whose frequency is close to the field frequency. Growth rates can be closely approximated by simple algebraic formulae, as can the critical magnetic field strength for the onset of instability.

\section{Introduction}

Many metallurgical processes use an alternating magnetic field to control the free surface of a liquid metal. The field induces current flow in the metal generating a $\boldsymbol{J} \times \boldsymbol{B}$ force which at high frequency penetrates only a narrow surface layer of the metal. The mean component of the force is then equivalent to a magnetic pressure which may be used to support or control the shape of a free surface. For example in electromagnetic casting of aluminium the liquid metal is supported below by metal already solidified, and laterally by high-frequency alternating fields. Other applications include electromagnetic levitation and cold crucible melting, and are reviewed by Sneyd (1993).

Stability of the free surface is important for the success of these applications. Striations sometimes form on the surface of electromagnetically cast aluminium ingots with the consequence that a surface layer must be shaved off, and it is believed that these are due to small-lengthscale MHD instabilities. One of the first theoretical studies, Sagardia (1974), assumes a perfectly conducting fluid, and concludes that the magnetic field is stabilizing. Garnier \& Moreau (1983) consider the stability of a plane interface separating an infinitely deep layer fluid of finite electrical conductivity from an insulating one carrying a uniform alternating magnetic field. Their analysis also allows for a difference in mean velocity between the layers, thereby including a Kelvin-Helmholtz instability. Garnier \& Moreau assume a high magnetic field frequency so the Lorentz force can be averaged over one cycle, and the conclusion again is that electromagnetic effects are stabilizing. However Deepak \& Evans (1995) point out that this result is somewhat misleading, and that an extension of the Garnier 
$\&$ Moreau (1983) analysis which does not entirely neglect the wave frequency $\Omega$ in comparison with the field frequency $\Omega$, predicts weak instability. The Deepak \& Evans (1995) paper is also based on the high-frequency assumption but includes viscous damping, which somewhat reduces the predicted growth rates. Ramos \& Castellanos (1996) consider the Garnier-Moreau problem for a layer of finite depth, so that reversing the sign of $g$ provides a stability analysis for magnetic levitation. They include viscosity but take $\Omega / \omega$ to be zero, thereby suppressing the weak magnetic instability. A surprising result is that a combination of magnetic and viscous effects may be destabilizing in magnetic levitation.

In each of the last three references the magnetic Reynolds number $R_{m}$ is assumed small so that magnetic field perturbations due to the induced current can be ignored - a reasonable assumption since in practice $R_{m}$ is of order $10^{-2}$. Nevertheless setting $R_{m}=0$ ignores the potentially important effect of ohmic damping which could well represent a more significant energy loss than viscous dissipation. One study which considers a finite $R_{m}$ is that of McHale \& Melcher (1982). The focus of this work is however on instability of the induced bulk fluid motion, and a large part of the work concerns a container with a rigid lid. Free surfaces are also considered but the results are not significantly affected. A review of stability studies related to electromagnetic processing of liquid metals has been written by Fautrelle (1991).

In the first part of this paper we reconsider the Garnier-Moreau problem, but without invoking the high field frequency approximation. In $\$ 2$ a new weak formulation is used to derive a free-surface evolution equation which takes the form of a harmonic oscillator forced by a periodic convolution integral. A method of determining growth rates using Floquet theory is developed in $\S 3$, and it is found that the high-frequency averaging method gives accurate results except for bands of wavelengths near a resonance point, at which the natural (unforced) wave frequency is an integral multiple of the forcing frequency. Near a resonance the growth rates increase typically by an order of magnitude and can be approximated quite accurately by a simple algebraic formula.

In $\S 4$ we extend the theory to include ohmic and viscous damping (assuming that both effects are relatively weak). It is found that viscous damping has little effect except for extremely short wavelengths, while ohmic dissipation completely damps all instabilities except those near the first resonance point. For a given frequency there is a critical magnetic field strength $B_{C}$ for the onset of instability, which is given by a simple approximate formula.

\section{Evolution equation}

A semi-infinite body of inviscid, incompressible, electrically conducting fluid, occupies the region $z<0$ (region 1). The $z$-axis is taken vertically upwards so the fluid surface is initially horizontal. In the space $z>0$ above the fluid (region 2) there is a vacuum (or relatively light insulating gas such as air) and a uniform magnetic field

$$
\boldsymbol{B}=B_{0} \cos (\omega t) \widehat{\boldsymbol{y}}
$$

is applied. Figure 1 shows a diagram of the system. This section is devoted to deriving an evolution equation for a free-surface perturbation

$$
z=\eta(x, y, t)
$$

on which we shall base our stability analysis. 


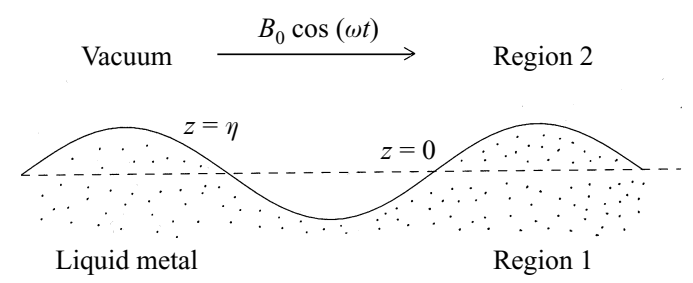

FIGURE 1. Diagram of the system.

The equations we use are Maxwell's equations, and Ohm's law for moving conductor,

$$
\nabla \times \boldsymbol{B}=\mu_{0} \boldsymbol{J}, \quad \frac{\partial \boldsymbol{B}}{\partial t}=-\nabla \times \boldsymbol{E}, \quad \boldsymbol{J}=\sigma(\boldsymbol{E}+\boldsymbol{v} \times \boldsymbol{B}),
$$

together with Euler's equations of motion for an incompressible fluid,

$$
\nabla \cdot \boldsymbol{v}=0, \quad \rho \frac{\partial \boldsymbol{v}}{\partial t}+\rho(\boldsymbol{v} \cdot \nabla) \boldsymbol{v}=-\rho g \widehat{z}-\nabla P_{T}+\frac{1}{\mu_{0}}(\boldsymbol{B} \cdot \nabla) \boldsymbol{B},
$$

where

$$
P_{T}=P+\frac{1}{2 \mu_{0}} B^{2}
$$

is the sum of the fluid and magnetic pressures. Here $\boldsymbol{B}, \boldsymbol{E}, \boldsymbol{J}$ represent the magnetic field, the electric field and the current density, and $\sigma$ the electrical conductivity of the medium; $\boldsymbol{v}$ is the fluid velocity, $\rho$ its density, and $g$ gravitational acceleration. Equations (2.2) combine to give the magnetic induction equation,

$$
\frac{\partial \boldsymbol{B}}{\partial t}=\nabla \times(\boldsymbol{v} \times \boldsymbol{B})+\lambda \nabla^{2} \boldsymbol{B}, \quad \lambda=1 /\left(\mu_{0} \sigma\right),
$$

We adopt the approximation made by both Garnier \& Moreau (1983) and Deepak \& Evans (1995) that the magnetic Reynolds number $R_{m}$ is small; consequently the induction term $\nabla \times(\boldsymbol{v} \times \boldsymbol{B})$ is neglected until $\S 4$ where we examine electromagnetic and viscous damping.

\subsection{Steady state}

In the steady state, the fluid velocity $\boldsymbol{v}=0$ and the free surface $z=0$ unperturbed. The magnetic field in the vacuum is given by (2.1) while that in the fluid is found by solving (2.5), which as a consequence of the small- $R_{m}$ assumption, reduces to

$$
\frac{\partial \boldsymbol{B}}{\partial t}=\lambda \nabla^{2} \boldsymbol{B} .
$$

Invoking continuity of $\boldsymbol{B}$ across the interface we obtain

$$
\boldsymbol{B}=B_{0} \mathrm{e}^{K z} \cos (\omega t+K z) \widehat{\boldsymbol{y}}, \quad K=(\omega / 2 \lambda)^{1 / 2}=1 / \delta,
$$

where $\delta$ represents the electromagnetic skin depth. The current in the fluid is given by

$$
\boldsymbol{J}=-\left(B^{\prime} / \mu_{0}\right) \widehat{\boldsymbol{x}},
$$

where the prime denotes differentiation with respect to $z$. Since $(\boldsymbol{B} \cdot \nabla) \boldsymbol{B}=0$ and $\boldsymbol{v}=0$ Euler's equation (2.3) simply gives

$$
P_{T}=P_{T 0}-\rho g z, \quad P_{T 0}=P_{0}+\frac{1}{2 \mu_{0}} B_{0}^{2} \cos ^{2}(\omega t),
$$

where $P_{0}$ is the (constant) pressure at the interface. 


\subsection{Perturbed state}

We now suppose the interface is perturbed to $z=\eta(x, y, t)$ say, making the usual linear wave theory assumption that the derivatives $\eta_{x}$ and $\eta_{y}$ are small. The displacement $\eta$ can be written as a Fourier integral,

$$
\eta(\boldsymbol{x}, t)=\frac{1}{4 \pi^{2}} \int_{-\infty}^{\infty} \hat{\eta}(\boldsymbol{k}, t) \mathrm{e}^{\mathrm{i} \boldsymbol{k} x} \mathrm{~d} \boldsymbol{k} .
$$

To follow the time evolution of $\eta$ we study that of a single Fourier component, say

$$
\eta=h(t) \mathrm{e}^{\mathrm{i} k x} .
$$

This displacement perturbs the magnetic field to $\boldsymbol{B}+\boldsymbol{b}$ say, and we can write the field perturbation in the form

$$
\boldsymbol{b}=\boldsymbol{f}(z, t) \mathrm{e}^{\mathrm{i} k \boldsymbol{x}} .
$$

In the non-conducting region $2, \nabla \times \boldsymbol{b}_{2}=0$, so there is a scalar potential $\psi(t, z) \mathrm{e}^{\mathrm{i} k x}$ for $\boldsymbol{b}_{2}$ which satisfies Laplace's equation. Since also $\boldsymbol{b}_{2} \rightarrow 0$ for large $z$ we find

$$
\boldsymbol{b}_{2}=\nabla\left[\psi_{0}(t) \mathrm{e}^{\mathrm{i} k x} \mathrm{e}^{-k z}\right],
$$

where $\psi_{0}(t)$ is an arbitrary function of time.

In the conducting region 1 , it turns out that we need calculate only the $z$-component of the field. Since $\boldsymbol{b}_{1}$ satisfies (2.6)

$$
\frac{\partial f_{1 z}}{\partial t}=\lambda\left(\frac{\partial^{2} f_{1 z}}{\partial z^{2}}-k^{2} f_{1 z}\right) .
$$

This may be solved by taking a Fourier transform in time, writing

$$
\hat{f}_{1 z}(z, \alpha)=\int_{-\infty}^{\infty} \mathrm{e}^{\mathrm{i} \alpha t} f_{1 z}(z, t) \mathrm{d} t,
$$

to obtain

$$
\hat{f}_{1 z}=\hat{f}_{1 z}(0, \alpha) \mathrm{e}^{\chi z}, \quad \chi(\alpha)=\left(k^{2}-\mathrm{i} \alpha / \lambda\right)^{1 / 2},
$$

where the square root branch is chosen so that the real part of $\chi$ is positive. To invoke continuity of magnetic field across the interface we must evaluate $\boldsymbol{B}+\boldsymbol{b}$ on $z=\eta$, using a Taylor series expansion of the first (zeroth-order) term

$$
\boldsymbol{f}_{1}(0, t)+h(t) \boldsymbol{B}^{\prime}(0, t)=\boldsymbol{f}_{2}(0, t)=\boldsymbol{H} \psi_{0}(t),
$$

where we have used (2.9) and defined

$$
\boldsymbol{H}=\left(\mathrm{i} k_{x}, \mathrm{i} k_{y},-k\right) .
$$

Using (2.11) and the fact that $\nabla \cdot \boldsymbol{b}_{1}=0$ we establish the boundary condition

$$
f_{1 z}^{\prime}(0, t)+k f_{1 z}(0, t)=\mathrm{i} h k_{y} B^{\prime}(0, t),
$$

which combined with (2.10) gives

$$
\hat{f}_{1 z}=-\frac{\mathrm{i} k_{y} K B_{0}}{k+\chi} \mathrm{e}^{\chi z} \hat{\zeta} .
$$

Here

$$
\zeta=(\cos \omega t-\sin \omega t) h(t), \quad \hat{\zeta}=\mathscr{F}_{t}\{\zeta\},
$$

where $\mathscr{F}_{t}$ denotes the operation of taking the Fourier transform in time. 
2.3. Evolution equation for $h(t)$

For small-amplitude perturbations, the Euler equation (2.3) takes the form

$$
\rho \frac{\partial \boldsymbol{v}}{\partial t}+\nabla p_{T}=\frac{1}{\mu_{0}}((\boldsymbol{b} \cdot \nabla) \boldsymbol{B}+(\boldsymbol{B} \cdot \nabla) \boldsymbol{b}),
$$

where $p_{T}$ is the perturbation total pressure.

In deriving an evolution equation for $h(t)$ our strategy is to use the 'weak solution' approach, obtaining information from inner products of (2.15) with suitable test functions. Specifically we define a test function,

$$
\phi=\frac{1}{2 \pi} \mathrm{e}^{-\mathrm{i} k x} \mathrm{e}^{k z}
$$

with the properties

$$
\nabla^{2} \phi=0, \quad \phi(x, 0)=\frac{1}{2 \pi} \mathrm{e}^{-\mathrm{i} k x}, \quad \phi_{z}(x, 0)=\frac{k}{2 \pi} \mathrm{e}^{-\mathrm{i} \boldsymbol{k} x}, \quad \lim _{z \rightarrow-\infty} \phi=0,
$$

and an inner product

$$
\langle\boldsymbol{F}, \nabla \phi\rangle=\int_{V} \boldsymbol{F} \cdot \nabla \phi \mathrm{d} V,
$$

where the volume of integration $V$ is defined to be a unit length of one wavelength interval, i.e. the region

$$
0 \leqslant \boldsymbol{k} \cdot \boldsymbol{x} \leqslant 2 \pi, \quad 0 \leqslant(\boldsymbol{k} \times \widehat{\boldsymbol{z}}) \cdot \boldsymbol{x} \leqslant k, \quad-\infty<z \leqslant 0 .
$$

We now calculate the inner products $I_{1}, I_{2}, I_{3}$ of $\nabla \phi$ with each of the three terms in (2.15). The first gives

$$
I_{1}=\rho \int_{V} \dot{\boldsymbol{v}} \cdot \nabla \phi \mathrm{d} V=\rho \int_{V} \nabla \cdot(\dot{\boldsymbol{v}} \phi) \mathrm{d} V=\rho \int_{\partial V} \phi \dot{\boldsymbol{v}} \cdot \mathrm{d} \boldsymbol{S},
$$

where $\partial V$ is the boundary of $V$. By periodicity of $\boldsymbol{v}$ and $\phi$, all contributions to the surface integral vanish, except that from the free surface $z=0$, which we denote by $S$. Applying the usual kinematic condition,

$$
\left(v_{z}\right)_{z=0}=\dot{\eta},
$$

we conclude that

$$
I_{1}=\rho \ddot{h} / k
$$

The pressure term in (2.15) gives

$$
I_{2}=\int_{V} \nabla p_{T} \cdot \nabla \phi \mathrm{d} V=\int_{V} \nabla \cdot\left(p_{T} \nabla \phi\right) \mathrm{d} V=\int_{S} p_{T} \nabla \phi \cdot \mathrm{d} \boldsymbol{S} .
$$

Again the only contribution is the integral over the free surface $S$, so

$$
I_{2}=\int_{S} p_{T} \phi_{z} \mathrm{~d} x \mathrm{~d} y=\frac{k}{2 \pi} \int_{S} p_{T} \mathrm{e}^{-\mathrm{i} \boldsymbol{k} x} \mathrm{~d} x \mathrm{~d} y .
$$

Because the fluid pressure and magnetic field are continuous across $z=\eta$, so is $P_{T}+p_{T}$ :

$$
P_{T 0}+\eta \frac{\partial P_{T}}{\partial z}+p_{T 1}+\gamma \nabla^{2} \eta=P_{T 0}+p_{T 2}
$$

where $\gamma$ is surface tension, and all terms are evaluated at $z=0$. Substituting from 
(2.8) we find

$$
p_{T 1}=\rho g \eta-\gamma \nabla^{2} \eta+\frac{B b_{y 2}}{\mu_{0}}
$$

and substituting (2.19) into (2.18) finally gives

$$
I_{2}=\left(\rho g+\gamma k^{2}+\right) h-\frac{i k_{y}}{\mu_{0} k} B f_{1 z} .
$$

In the last term we have used (2.11) to re-express $f_{2 y}$ in terms of $f_{1 z}$.

The inner product from the right-hand side of $(2.15)$ is

$$
I_{3}=-\frac{1}{k \mu_{0}} \int_{-\infty}^{0} \boldsymbol{H} \cdot\left[\mathrm{i} k_{y} B \boldsymbol{f}+f_{z} B^{\prime} \hat{\boldsymbol{y}}\right] \mathrm{e}^{k z} \mathrm{~d} z
$$

which by means of the identity $\mathrm{i} k_{x} f_{x}+\mathrm{i} k_{y} f_{y}+f_{z}^{\prime}=0$ simplifies to

$$
I_{3}=\frac{1}{k \mu_{0}} \int_{-\infty}^{0}\left[\mathrm{i} k_{y} B\left(f_{z} \mathrm{e}^{k z}\right)^{\prime}-\mathrm{i} k_{y} B^{\prime} f_{z} \mathrm{e}^{k z}\right] \mathrm{d} z .
$$

Integrating the final term by parts we obtain

$$
I_{3}=\frac{2 \mathrm{i} k_{y}}{k \mu_{0}} \int_{-\infty}^{0} B\left(f_{z} \mathrm{e}^{k z}\right)^{\prime} \mathrm{d} z-\frac{\mathrm{i} k_{y}}{\mu_{0} k} B f_{1 z} .
$$

The evolution equation is derived by substituting (2.17), (2.20), and (2.21) into $I_{1}+I_{2}=I_{3}$ :

$$
\ddot{h}+\Omega^{2} h=\frac{2 \mathrm{i} k_{y}}{\rho \mu_{0}} \int_{-\infty}^{0} B\left(f_{z} \mathrm{e}^{k z}\right)^{\prime} \mathrm{d} z,
$$

where $\Omega=\left(g k+\gamma k^{3} / \rho\right)^{1 / 2}$ is the frequency of a gravity-capillary wave of wavenumber $k$.

The final step is to express the integral, say $I_{M}$, on the right-hand side of (2.22) in terms of $h$. Substituting for $B$ and $f_{z}$ from (2.7) and (2.13) we find

$$
I_{M}=C k \int_{-\infty}^{0} \mathrm{e}^{(k+K) z} \cos (\omega t+K z) \mathscr{F}_{t}^{-1}\left\{\hat{\zeta}^{\chi z}\right\} \mathrm{d} z,
$$

where the constant

$$
C=\frac{-2 k_{y}^{2} B_{0}^{2} K}{k \rho \mu_{0}}
$$

and $\mathscr{F}_{t}^{-1}$ represents an inverse Fourier transform with respect to $t$. The $z$-integration can be carried out explicitly to give

$$
I_{M}=\frac{C}{4}\left[\left(a_{0} * h\right)(t)+\mathrm{e}^{2 \mathrm{i} \omega t}\left(a_{1} * h\right)(t)+\mathrm{e}^{-2 \mathrm{i} \omega t}\left(a_{-1} * h\right)(t)\right],
$$

where the operation * represents the convolution product. The functions $a_{0}(t), a_{1}(t)$ and $a_{-1}(t)$ are most conveniently defined by their Fourier transforms with respect to $t$ :

$$
\begin{gathered}
\hat{a}_{0}(\alpha)=\frac{k(1-\mathrm{i})}{\kappa+\chi(\alpha+\omega)}+\frac{k(1+\mathrm{i})}{\kappa^{*}+\chi(\alpha-\omega)}, \\
\hat{a}_{1}(\alpha)=\frac{k(1+\mathrm{i})}{\kappa+\chi(\alpha-\omega)}, \quad \hat{a}_{-1}(\alpha)=\frac{k(1-\mathrm{i})}{\kappa^{*}+\chi(\alpha+\omega)},
\end{gathered}
$$

where $\kappa=k+K(1+\mathrm{i})$ and the ${ }^{*}$ denotes a complex conjugate. 
The final form of the evolution equation (2.22) is

$$
\ddot{h}+\Omega^{2} h+\Omega^{2} M \Gamma \int_{-\infty}^{\infty}\left[a_{0}(\xi)+a_{1}(\xi) \mathrm{e}^{2 \mathrm{i} \omega t}+a_{-1}(\xi) \mathrm{e}^{-2 \mathrm{i} \omega t}\right] h(t-\xi) \mathrm{d} \xi=0 .
$$

Here $M$ and $\Gamma$ are dimensionless numbers measuring the strength of the magnetic field, and the relative importance of surface tension respectively:

$$
M=\frac{B_{0}^{2} \cos ^{2} \theta}{2 \mu_{0} \rho g \delta}, \quad \Gamma=\frac{g k}{\Omega^{2}},
$$

where $\theta$ is the angle between the wavenumber vector $\boldsymbol{k}$ and the applied magnetic field.

\section{Stability analysis}

\subsection{Theoretical considerations}

The stability of the system depends on whether there exist growing solutions of (2.25). This is a kind of generalized Mathieu-Hill type equation and the standard results of Floquet Theory (see e.g. Jordan \& Smith 1987) would be valid, provided we could establish the existence of a finite fundamental set of solutions of (2.25). However, this matter is not easily settled and Sneyd (1996) gives an example of an equation of the form

$$
\ddot{h}+\Omega^{2} h=\int_{-\infty}^{\infty} f(\xi) h(t-\xi) \mathrm{d} \xi
$$

which has infinitely many independent solutions. Such behaviour is however associated with a singularity in the kernel function $f(\xi)$ at infinity, which is not the case with (2.25).

Assuming for the moment that a finite fundamental set of solutions of (2.25) exists, then Floquet theory shows that these solutions can be taken to be of the form

$$
h(t)=\mathrm{e}^{\mathrm{i} s t} P(t),
$$

where $P(t)$ is periodic with period $\pi / \omega$. If, for one of the fundamental solutions the imaginary part of $s$ is negative, then the system is unstable. We proceed by assuming solutions in the form (3.1). Indeed, quite aside from the question of whether or not there is a finite fundamental set of solutions, if we can demonstrate the existence of solutions of the form (3.1) with $\operatorname{Im}(s)<0$ the system must be unstable, but the largest growth rate found represents only a lower bound, i.e. there may exist more rapidly growing solutions of a different form.

\subsection{High-frequency limit}

Both Garnier \& Moreau (1983) and Deepak \& Evans (1995) make a high-fieldfrequency approximation, assuming $\omega \gg \Omega$. Averaging (2.25) over one field period $\pi / \omega$ eliminates the final two terms, to give

$$
\ddot{h}+\Omega^{2} h=\Omega^{2} M \Gamma \int_{-\infty}^{\infty} a_{0}(\xi) h(t-\xi) \mathrm{d} \xi .
$$

Taking a Fourier transform of (3.2) yields

$$
\hat{h}\left[\Omega^{2}-\alpha^{2}+\epsilon \Omega^{2} \hat{a}_{0}(\alpha)\right]=\hat{h} f(\alpha) \quad \text { say } \quad=0,
$$


whose solution is

$$
\hat{h}=c_{1} \delta\left(\alpha-\alpha_{1}\right)+c_{2} \delta\left(\alpha-\alpha_{2}\right),
$$

where the $c_{i}$ are arbitrary constants and the $\alpha_{i}$ are the zeros of $f(\alpha)$. The Fourier inverse of this solution is

$$
h=c_{1} \mathrm{e}^{-\mathrm{i} \alpha_{1} t}+c_{2} \mathrm{e}^{-\mathrm{i} \alpha_{2} t}
$$

so the instability growth rate can be estimated from the imaginary parts of the $\alpha_{i}$. It can be shown (after a little algebra) that the equation $f(\alpha)=0$ is identical to the dispersion relation (38) in Garnier \& Moreau (1983), provided we set $\boldsymbol{U}_{1}=\boldsymbol{U}_{2}=0$, $\rho_{2}=0$ and evaluate $\hat{a}_{0}$ at $\alpha=0$.

Finding the $\alpha_{i}$ involves solving a transcendental equation, but if we assume $\epsilon$ small, then to leading order,

$$
\alpha_{1}=\Omega+\frac{1}{2} \epsilon \Omega \hat{a}_{0}(\Omega), \quad \alpha_{2}=-\Omega-\frac{1}{2} \epsilon \Omega \hat{a}_{0}(-\Omega) \quad(\epsilon=M \Gamma) .
$$

It can be seen from $(2.23)$ that $\hat{a}_{0}(-\Omega)=\hat{a}_{0}^{*}(\Omega)$ (where the $*$ denotes the complex conjugate) so the imaginary parts of the two solutions are equal. The approximate growth rate $\sigma$ say is therefore given by

$$
\sigma=\frac{1}{2} \Omega \epsilon \operatorname{Im}\left\{\hat{a}_{0}(\Omega)\right\} .
$$

It is pointed out by Deepak \& Evans (1995) that Garnier \& Moreau (1983) made the further approximation of setting $\Omega=0$ in (3.3), which yields $\sigma=0$ since $\hat{a}_{0}(0)$ is real. This explains their conclusion that alternating field is not destabilizing. Without this approximation the conclusion is just the opposite; in all cases we find $\sigma>0$, although often the growth rates are quite small.

Interestingly, (3.3) can be derived for all frequencies when the field is relatively weak, i.e. $\epsilon$ small. According to Floquet theory the stability of solutions of (2.25) is determined by calculating the Floquet matrix, $\boldsymbol{F}$ say, which advances the solution by one period $T=\pi / \omega$. In other words, if $h(t)$ is a solution of (2.25) then

$$
\left(\begin{array}{l}
h(t+T) \\
\dot{h}(t+T)
\end{array}\right)=\boldsymbol{F}\left(\begin{array}{l}
h(t) \\
\dot{h}(t)
\end{array}\right)
$$

for all time $t$.

The Floquet matrix is readily calculated to order $\epsilon$ using methods developed in Sneyd (1996), and one obtains

$$
\boldsymbol{F}=\left(\begin{array}{cc}
\mathrm{e}^{\mathrm{i} \Omega T} & 0 \\
0 & \mathrm{e}^{-\mathrm{i} \Omega T}
\end{array}\right)+\frac{1}{2} \mathrm{i} \epsilon \Omega\left(\begin{array}{cc}
f_{1} & f_{2} \\
-f_{2}^{*} & -f_{1}^{*}
\end{array}\right),
$$

where

$$
f_{1}=\hat{a}_{0}(-\Omega) T \mathrm{e}^{\mathrm{i} \Omega T}, \quad f_{2}=2 \sin \Omega T\left[\frac{\hat{a}_{0}(\Omega)}{2 \Omega}+\frac{\hat{a}_{1}(\Omega)}{2 \Omega-\omega}+\frac{\hat{a}_{-1}(\Omega)}{2 \Omega+\omega}\right] .
$$

To first order in $\epsilon$ the eigenvalues of $\boldsymbol{F}$ are

$$
\lambda_{i}=\mathrm{e}^{ \pm \mathrm{i} \Omega T}\left[1 \pm \frac{1}{2} \mathrm{i} \epsilon \Omega T \hat{a}_{0}(\mp \Omega)\right] .
$$

The two eigenvalues give identical growth rates

$$
\sigma=T^{-1}\left|\lambda_{i}-1\right|=\frac{1}{2} \Omega \epsilon \operatorname{Im}\left\{\hat{a}_{0}(\Omega)\right\},
$$

which is the same estimate as (3.3). 


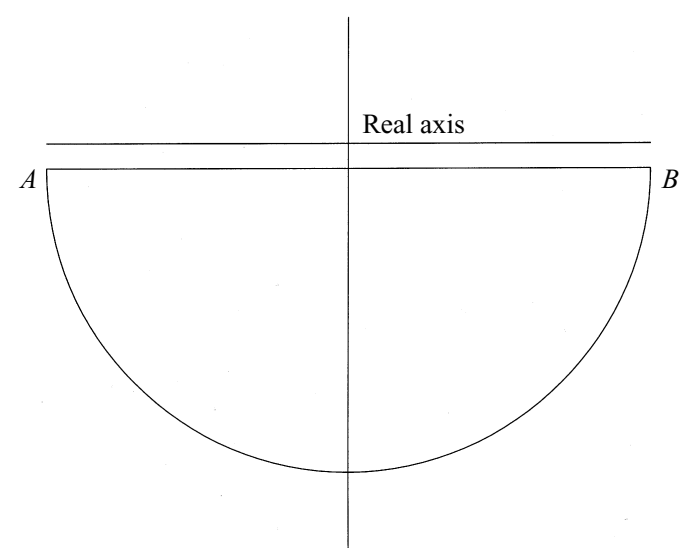

FIgURe 2. Contour $\mathscr{C}$ in the complex plane.

\subsection{General method of analysis}

Assuming a solution of the form (3.1) we write

$$
h(t)=\mathrm{e}^{\mathrm{i} s t} \sum_{n=-N}^{n=N} \alpha_{n} \mathrm{e}^{\mathrm{in} n \omega t}=\sum_{n=-N}^{n=N} \alpha_{n} \mathrm{e}^{\mathrm{is}_{n} t} \quad \text { say, }
$$

where $s_{n}=s+2 n \omega$ and $N$ represents a suitable truncation level for the Fourier series. Substitution into (2.25) and equating Fourier coefficients gives

$$
\left[\Omega^{2}-s_{n}^{2}\right] \alpha_{n}+\Omega^{2} \epsilon\left[\alpha_{n} \hat{a}_{0}\left(-s_{n}\right)+\alpha_{n-1} \hat{a}_{1}\left(-s_{n}\right)+\alpha_{n+1} \hat{a}_{-1}\left(-s_{n}\right)\right]=0 .
$$

This system of equations can be written in the form

$$
\boldsymbol{A} \boldsymbol{\alpha}=0, \quad \boldsymbol{\alpha}^{T}=\left(\alpha_{N}, \alpha_{N-1}, \ldots, \alpha_{-N}\right),
$$

where $\boldsymbol{A}$ is a tridiagonal matrix, the three elements along row $n$ being,

$$
\begin{gathered}
\Omega^{2} \epsilon \hat{a}_{1}\left(-s_{n}\right), \\
\Omega^{2}-s_{n}^{2}+\Omega^{2} \epsilon \hat{a}_{0}\left(-s_{n}\right), \\
\Omega^{2} \epsilon \hat{a}_{-1}\left(-s_{n}\right),
\end{gathered}
$$

The condition for this system to have a non-trivial solution for $\alpha$ is

$$
\operatorname{det}(\boldsymbol{A})=F(s)=0 .
$$

Instability growth rates are therefore obtained as zeros of $F(s)$. Note that if we take $N=0$, we recover the equation $f(s)=0$ for the high-frequency approximation discussed above.

Equation (3.4) turns out to be somewhat difficult to solve numerically. Various methods were tried but the most reliable was based on the well-known result

$$
\Delta_{\mathscr{C}} \arg f(z)=2 \pi(N-P)
$$

for the change in argument of an analytic function around a closed contour $\mathscr{C}$, see e.g. Titchmarsh $(1939, \S 3.4)$. Here $N$ is the number of zeros and $P$ the number of poles of $f(z)$ inside $\mathscr{C}$. The chosen contour is shown in figure 2 , and consists of the straight line segment $\operatorname{Im}(s)=b$, completed by a large semi-circle in the lower half of the complex plane. 


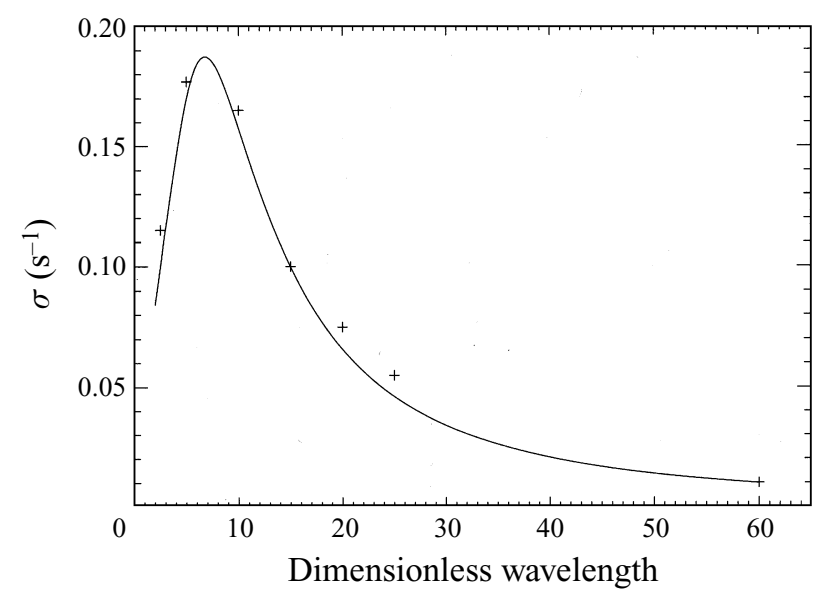

FIgURE 3. Comparison with results of Deepak $\&$ Evans (1995) (crosses). $\delta=0.0081 \mathrm{~m}$,

$$
f=6283.2 \mathrm{~Hz}, \epsilon=10.9 \text {. }
$$

Since $F(s) \approx s^{4 N+2}$ as $|s| \rightarrow \infty$, the change in argument of $F(s)$ around the semicircle $A B$ is $(4 N+2) \pi$. The number $n(b)$ of zeros of $F(s)$ lying below the line $A B$ is therefore given by

$$
n_{b}=2 N+1-\Delta_{A B} \arg (F(s)) .
$$

The change in argument in $F(s)$ is readily calculated by stepping along $A B$, but if this line passes close to a zero the argument may change rapidly. Thus it was found necessary control the step length to prevent any increment in the argument exceeding 0.2 radians. A bisection method was used to locate the value of $b$, say $b_{c}$, at which $n_{b}$ becomes zero; $b_{c}$ then equals the largest negative imaginary part of any zero, and hence the growth rate of the instability.

To check the method figure 3 shows a comparison of our results with those of Deepak \& Evans (1995). The solid line represents our calculations and the crosses selected points on the graph $(b)$ in Deepak \& Evans (1995) figure 4. It can be seen that the agreement is quite good, given that the comparison points were estimated from a rather small graph.

Typical results are shown in figure 4 as graphs of growth rate versus $k$ for a given applied frequency $\omega$ and dimensionless field strength $\epsilon$. In all cases we took $\rho=2 \times 10^{3}$ and $\gamma=0.7$. For comparison the growth rate predicted by the highfrequency approximation (3.3) is shown as a dashed line. The most remarkable feature of the graphs is a series of sharp peaks in the growth rate, which occur near resonance points where the gravity-capillary frequency is an integral multiple of the applied frequency:

$$
\Omega=m \omega, \quad m=1,2,3, \ldots .
$$

(The positions of the resonances are marked by vertical dotted lines.) Even at the relatively low frequency of $8 \mathrm{~Hz}$ the approximation is remarkably accurate, except near the resonances, and improves (as expected) as the frequency increases.

In order to understand the growth-rate peak near the resonance points we assume that $\epsilon$ is small. To leading order in $\epsilon$ the matrix $\boldsymbol{A}$ is diagonal, and the zeros are given by

$$
s=-2 n \omega \pm \Omega, \quad-N \leqslant n \leqslant N .
$$


Two zeros corresponding to $n=n_{1}, n=n_{2}$ are equal if

$$
\Omega=\left(n_{1}-n_{2}\right) \omega,
$$

i.e. if we are at a resonance point. It appears therefore that the imaginary part of nearly coincident zeros grows very rapidly in the vicinity of a resonance point, but is relatively small everywhere else.

To demonstrate this we truncate the system of equations to $N=1$ so that we are dealing with a $3 \times 3$ matrix $\boldsymbol{A}$. The diagonal elements written to leading order and in dimensionless form are

$$
\left(1+s^{\prime}-2 w\right)\left(1-s^{\prime}+2 w\right), \quad\left(1+s^{\prime}\right)\left(1-s^{\prime}\right) \quad\left(1+s^{\prime}+2 w\right)\left(1-s^{\prime}-2 w\right),
$$

where $s^{\prime}=s / \Omega$ and $w=\omega / \Omega$. At the first resonance $w=1$ the zeros $s^{\prime}= \pm 1$ are repeated, and we consider in detail the behaviour of the former zero in this neighbourhood, writing

$$
s^{\prime}=1+\epsilon \delta_{s}, \quad w=1+\epsilon \delta_{w} .
$$

The equation $\operatorname{det}(\boldsymbol{A})=0$ can now be written to first order in $\epsilon$ in the form

$$
\left|\begin{array}{lcr}
2 \epsilon\left(\delta_{s}-2 \delta_{w}\right)+\epsilon \hat{a}_{0}(\Omega) & \epsilon \hat{a}_{-1}(\Omega) & 0 \\
\epsilon \hat{a}_{1}(-\Omega) & -2 \epsilon \delta_{s}+\epsilon \hat{a}_{0}(-\Omega) & \epsilon \hat{a}_{-1}(-\Omega) \\
0 & \epsilon \hat{a}_{1}(-3 \Omega) & -8
\end{array}\right|=0 .
$$

Dividing the first two rows of the determinant by $\epsilon$ and then letting $\epsilon \rightarrow 0$ we find that $\delta_{s}$ satisfies a quadratic with solution,

$$
\delta_{s}=\delta_{w}-\frac{1}{2} \mathrm{i} \hat{a}_{0 I} \pm \frac{1}{2}\left[\left(2 \delta_{w}-\hat{a}_{0 R}\right)^{2}-\left|\hat{a}_{-1}\right|^{2}\right]^{1 / 2},
$$

where the $\hat{a}_{i}$ coefficients are all evaluated at $\Omega$ and the subscripts $R, I$ indicate real and imaginary parts. The corresponding growth rate $-\epsilon \operatorname{Im}\left(\delta_{s}\right)$ is also shown as a dashed curve in figure 4. Clearly, the growth rate corresponding to this zero is usually negative, but increases rapidly near a resonance point, outstripping the growth rates yielded by the other zeros. Equation (3.5) shows that maximum growth rate occurs at $\delta_{w}=-\hat{a}_{0 R} / 2$, a point lying slightly to the left of the resonance point. A possible interpretation of this result is that the presence of the magnetic field modifies usual gravity-capillary dispersion relation so that

$$
\Omega \rightarrow \Omega\left(1-\epsilon \hat{a}_{0 R} / 2\right) .
$$

The graphs in figure 4 show that as $\epsilon$ becomes smaller, the resonance point and point of maximum growth rate tend towards coinciding, as expected. The maximum growth rate can also be predicted from (3.5) to be $\left(\left|\hat{a}_{1}\right|^{2}-\hat{a}_{0 I}\right) / 2$. This is much larger than typical values away from the resonance point, since the real parts of the $\hat{a}_{i}$ coefficients are generally much larger than the imaginary parts (cf. (3.3)).

It is easily verified that the other repeated zero $s^{\prime}=-1$ gives an identical growth rate. The behaviour at other resonance points can be described by quadratic equations using similar methods, but the algebra becomes a lot more complicated, since to deal with the resonance $\Omega=n \omega$ one needs to consider a $(2 n+1) \times(2 n+1)$ determinant.

We have presented exact results for only relatively low-frequency fields. At higher frequencies the simple approximation (3.3) gives very good results, and resonances are less of an issue since they occur at high frequencies for short-wavelength waves which will be heavily damped by viscosity and ohmic dissipation - the subject of the next section. 

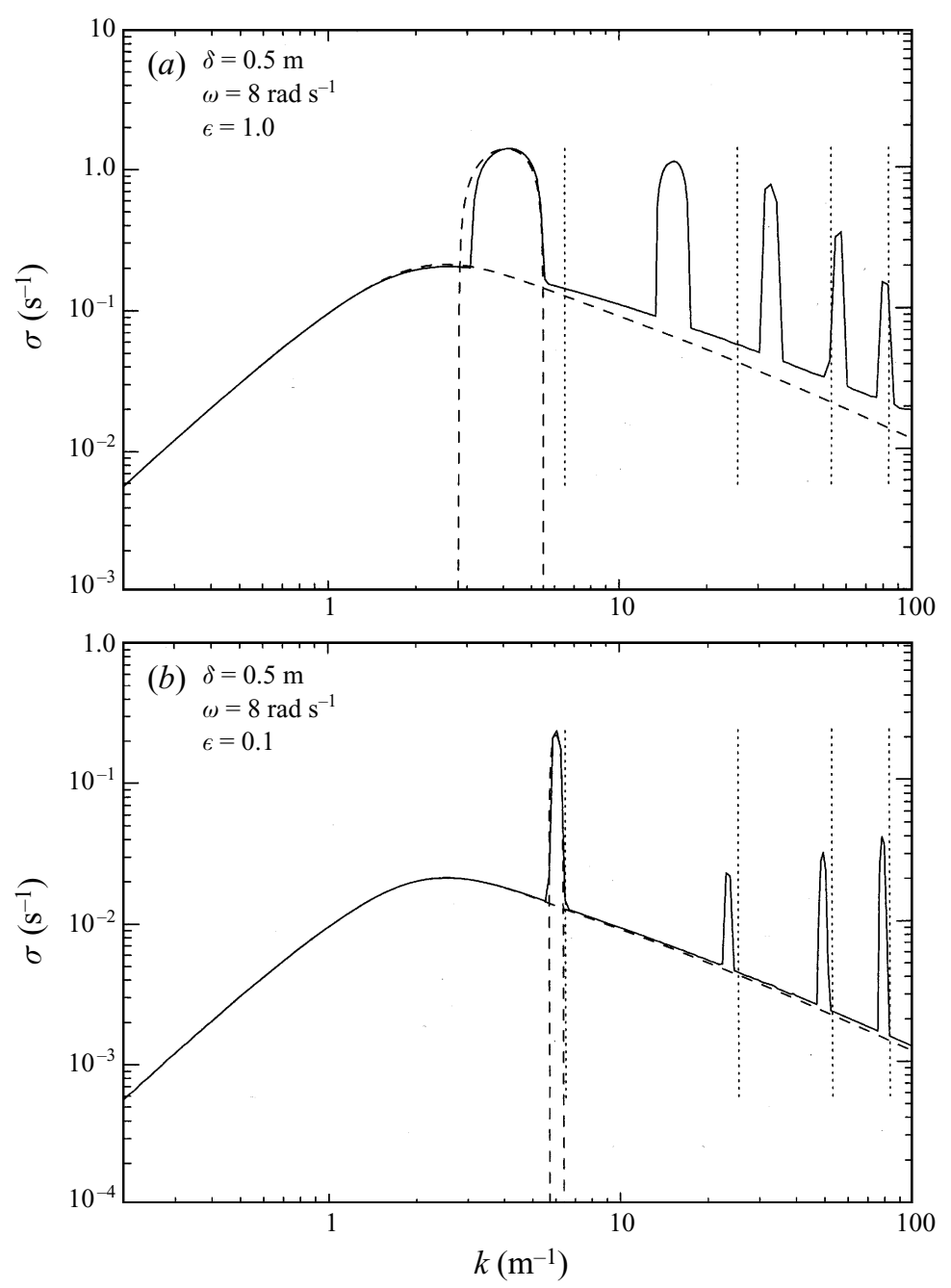

FiguRE $4(a, b)$. For caption see facing page.

\section{Damping}

There are two mechanisms for energy loss which we expect to somewhat reduce the growth rates calculated in the previous section - viscosity and ohmic dissipation. To obtain a tractable problem we assume that the flow associated with the passage of the wave approximates that of a simple gravity-capillary wave, i.e. that magnetic and viscous forces are relatively weak. To make this condition more precise we can write

$$
\boldsymbol{v}=\boldsymbol{v}_{P}+\boldsymbol{v}_{R},
$$

where the first term is the purely potential flow which would be associated with the wave in the absence of rotational forces, while the second is the magnetically-driven rotational component. The magnitude of $v_{R}$ can be estimated by taking the curl of (2.15), and $v_{P}=O(\Omega h)$. The ratio

$$
v_{R} / v_{P}=O(\epsilon)
$$



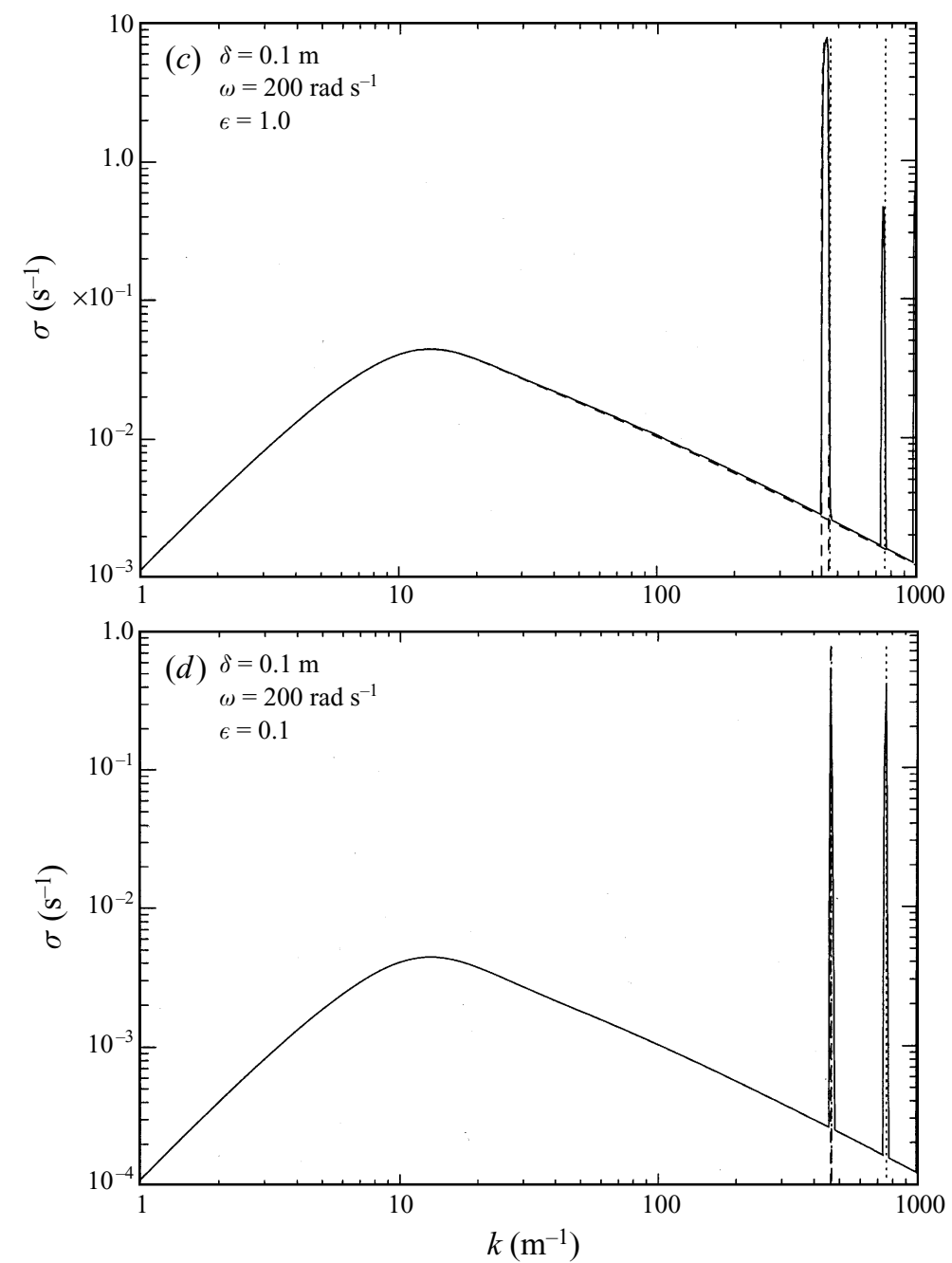

FIGURE $4(a-d)$. Graphs of growth rate $\sigma$ versus wavenumber $k$. The dashed lines plot the non-resonant approximations (3.3) and (3.5).

(cf. (2.25)) and our analysis therefore depends crucially on the assumption $\epsilon \ll 1$. This assumption will be valid for most industrial applications.

\subsection{Viscosity}

Including viscosity brings two changes to our previous equations. The first is the addition of a viscous term to our fluid motion equation (2.15) which becomes

$$
\rho \frac{\partial \boldsymbol{v}}{\partial t}+\nabla P=\boldsymbol{j} \times \boldsymbol{B}+\boldsymbol{J} \times \boldsymbol{b}+\mu \nabla^{2} \boldsymbol{v},
$$

where $\mu$ is dynamic viscosity. The second is to the boundary conditions at the free surface: in addition to the usual kinematic condition, zero stress at free surface implies

$$
w_{x}+u_{z}=0, \quad-P+2 \mu w_{z}=\gamma \eta_{x x},
$$

where for simplicity we consider a wave travelling in the $x$-direction, with wavenumber $(k, 0,0), \boldsymbol{v}=(u, 0, w)$, and the subscripts $x$ and $z$ represent partial derivatives. (Any 
viscous modification to the evolution equation must of course be independent of the direction of $\boldsymbol{k}$.)

The first extra term in the evolution equation for $h(t)$ arises from the inner product of our test function $\nabla \phi$ with the viscous term in (4.1). This can be written

$$
-\mu \int_{V}(\nabla \times \xi) \cdot(\nabla \phi) \mathrm{d} V=\mu \int_{V} \nabla \cdot(\nabla \phi \times \xi) \mathrm{d} V
$$

where $\xi=\left(u_{z}-w_{x}\right) \widehat{\boldsymbol{y}}$ is vorticity. Using the divergence theorem, the first of (4.2) and the kinematic free-surface condition (2.16), the last integral reduces to

$$
-2 \mu \int_{S}\left(k^{2} \eta_{t} \phi\right) \mathrm{d} S=-2 k \mu \dot{h} .
$$

The viscous boundary condition modifies the pressure term in the evolution equation. The second of (4.2) shows that the pressure perturbation on the free surface $(p)_{z=0}$ contains an extra term $2 \mu w_{z}$. At this point we use our assumption that fluid velocity differs only slightly from that of a simple gravity-capillary wave, and replace $w_{z}$ by $k w=k \dot{\eta}$. Substitution into (2.18) gives an extra term $2 k \mu \dot{h}$. In deriving the evolution equation (2.22) each of the inner products is multiplied by $k / \rho$, so the final effect of this contribution and that from (4.3) is to add the term

$$
4 v k^{2} \dot{h}
$$

to the left-hand side of (2.25) where $v$ is kinematic viscosity. (An equivalent result is derived in $\$ 348$ of Lamb 1932.)

Including the term (4.4) in the evolution equation (2.25) and time averaging as in $\$ 3.2$ we find that the growth rates are the zeros of

$$
\Omega^{2}+4 \mathrm{i} \alpha v k^{2}-\alpha^{2}+\epsilon \Omega^{2} \hat{a}_{0}(0)=0 .
$$

This equation is similar to (43) in Ramos \& Castellanos (1996), but does not include the mixed visco-magnetic term because of our weak-field approximation.

\subsection{Ohmic damping}

The physical basis of this damping mechanism is ohmic dissipation due to electric currents induced by the fluid motion. We need therefore to consider the full induction equation (2.5), which for the field perturbation is

$$
\frac{\partial \boldsymbol{b}}{\partial t}=\nabla \times(\boldsymbol{v} \times \boldsymbol{B})+\lambda \nabla^{2} \boldsymbol{b} .
$$

The magnetic term in the evolution equation is still given by (2.22) since only the solenoidal condition $\nabla \cdot \boldsymbol{b}=0$ and the boundary condition (2.12) were used in the derivation, so we need to calculate only the $z$-component of $\boldsymbol{b}$ or $f_{z}$.

Assuming the flow to approximate that due to an irrotational wave we can write

$$
\boldsymbol{v}=\nabla V, \quad V=\dot{h} k^{-1} \mathrm{e}^{\mathrm{i} k x} \mathrm{e}^{k z},
$$

and substitution into (4.5) produces

$$
\frac{\partial f_{z}}{\partial t}=\mathrm{i} k_{y} B \dot{h} \mathrm{e}^{k z}+\lambda\left(f_{z}^{\prime \prime}-k^{2} f_{z}\right)
$$

Substituting from (2.7) and taking a Fourier transform in time yields

$$
\hat{f}_{z}^{\prime \prime}-\chi^{2} \hat{f}_{z}=-c_{0}\left[\mathrm{e}^{\kappa z} \hat{\zeta}_{+}+\mathrm{e}^{\kappa^{*} z} \hat{\zeta}_{-}\right],
$$


where

$$
c_{0}=\frac{\mathrm{i} k_{y} B_{0}}{2 \lambda}, \quad \hat{\zeta}_{ \pm}=\mathscr{F}_{t}\left\{\mathrm{e}^{ \pm \mathrm{i} \omega t} \dot{h}\right\} .
$$

The differential equation (4.6), the Fourier transform of (2.12), and the condition that $f_{z}$ vanish as $z \rightarrow \infty$ are used to determine $f_{z}$. The expression $f_{z}^{\prime}+k f_{z}=f$ say, which appears in (2.22) is given by

$$
\hat{f}=\mathrm{i} K k_{y} B_{0} \hat{\mathrm{e}^{\chi z}}+\frac{c_{0}(\kappa+k)}{\kappa^{2}-\chi^{2}}\left(\mathrm{e}^{\chi z}-\mathrm{e}^{\kappa z}\right) \hat{\zeta}_{+}+\frac{c_{0}\left(\kappa^{*}+k\right)}{\kappa^{* 2}-\chi^{2}}\left(\mathrm{e}^{\chi z}-\mathrm{e}^{\kappa^{*} z}\right) \hat{\zeta}_{-} .
$$

Now this solution for $f$ is substituted into (2.22) to determine the new form of the magnetic term. The first term in (4.7) simply reproduces the magnetic term in (2.25). To evaluate second and third terms we perform the $z$-integration explicitly and invert the Fourier transform in terms of a convolution product to obtain

$$
{ }_{\epsilon} C_{D} \Omega \int_{-\infty}^{\infty}\left[d_{0}(\xi)+d_{1}(\xi) \mathrm{e}^{2 \mathrm{i} \omega t}+d_{-1}(\xi) \mathrm{e}^{-2 \mathrm{i} \omega t}\right] \dot{h}(t-\xi) \mathrm{d} \xi,
$$

where

and

$$
\begin{gathered}
\hat{d}_{0}(\alpha)=\frac{\mathrm{i} \beta_{1}}{\kappa+\chi(\alpha-\omega)}-\frac{\mathrm{i} \beta_{1}}{\kappa^{*}+\chi(\alpha-\omega)}+\frac{\mathrm{i} \beta_{1}^{*}}{\kappa+\chi(\alpha+\omega)}-\frac{\mathrm{i} \beta_{1}^{*}}{\kappa^{*}+\chi(\alpha+\omega)}, \\
\hat{d}_{1}(\alpha)=\frac{\beta_{2}}{[\kappa+\chi(\alpha-\omega)]^{2}}, \quad \hat{d}_{-1}(\alpha)=\frac{\beta_{2}^{*}}{\left[\kappa^{*}+\chi(\alpha+\omega)\right]^{2}}
\end{gathered}
$$

$$
\beta_{1}=\frac{k^{2}(k+\kappa)}{2 K(k+K)}, \quad \beta_{2}=1+k / \kappa, \quad C_{D}=\frac{\Omega \delta}{k \lambda} .
$$

\subsection{Evolution equation}

When the viscous damping term (4.4) and the magnetic damping term (4.8) are now included in the evolution equation (2.25) we find

$$
\begin{array}{r}
\ddot{h}+4 v k^{2} \dot{h}+\Omega^{2} h+\epsilon \int_{-\infty}^{\infty}\left[a_{0}(\xi)+a_{1}(\xi) \mathrm{e}^{2 \mathrm{i} \omega t}+a_{-1}(\xi) \mathrm{e}^{-2 \mathrm{i} \omega t}\right] h(t-\xi) \mathrm{d} \xi \\
+\epsilon C_{D} \int_{-\infty}^{\infty}\left[d_{0}(\xi)+d_{1}(\xi) \mathrm{e}^{2 \mathrm{i} \omega t}+d_{-1}(\xi) \mathrm{e}^{-2 \mathrm{i} \omega t}\right] \dot{h}(t-\xi) \mathrm{d} \xi=0 .
\end{array}
$$

As a first approximation we can perform the high-frequency analysis described in $\S 2.2$, and obtain growth rates given by

$$
\sigma=\frac{1}{2} \epsilon \Omega \operatorname{Im}\left\{\hat{a}_{0}(\Omega)\right\}-2 v k^{2}-\frac{1}{2} \epsilon C_{D} \Omega \operatorname{Re}\left\{\hat{d}_{0}(\Omega)\right\} .
$$

Also the exact method of $\$ 2.3$ can be applied to (4.9). As before growth rates are determined by solving

$$
\operatorname{det}(\boldsymbol{A})=F(s)=0,
$$

where $\boldsymbol{A}$ is a tridiagonal matrix, the three elements along row $n$ being

$$
\begin{gathered}
\Omega \epsilon\left[\Omega \hat{a}_{1}\left(-s_{n}\right)+\mathrm{i} C_{D} s_{n} \hat{d}_{1}\left(-s_{n}\right)\right], \\
\Omega^{2}-s_{n}^{2}+4 \mathrm{i} v k^{2} s_{n}+\Omega \epsilon\left[\Omega \hat{a}_{0}\left(-s_{n}\right)+\mathrm{i} C_{D} s_{n} \hat{d}_{0}\left(-s_{n}\right)\right], \\
\Omega \epsilon\left[\Omega \hat{a}_{-1}\left(-s_{n}\right)+\mathrm{i} C_{D} s_{n} \hat{d}_{-1}\left(-s_{n}\right)\right],
\end{gathered}
$$

where $s_{n}=s+2 n \omega$. 

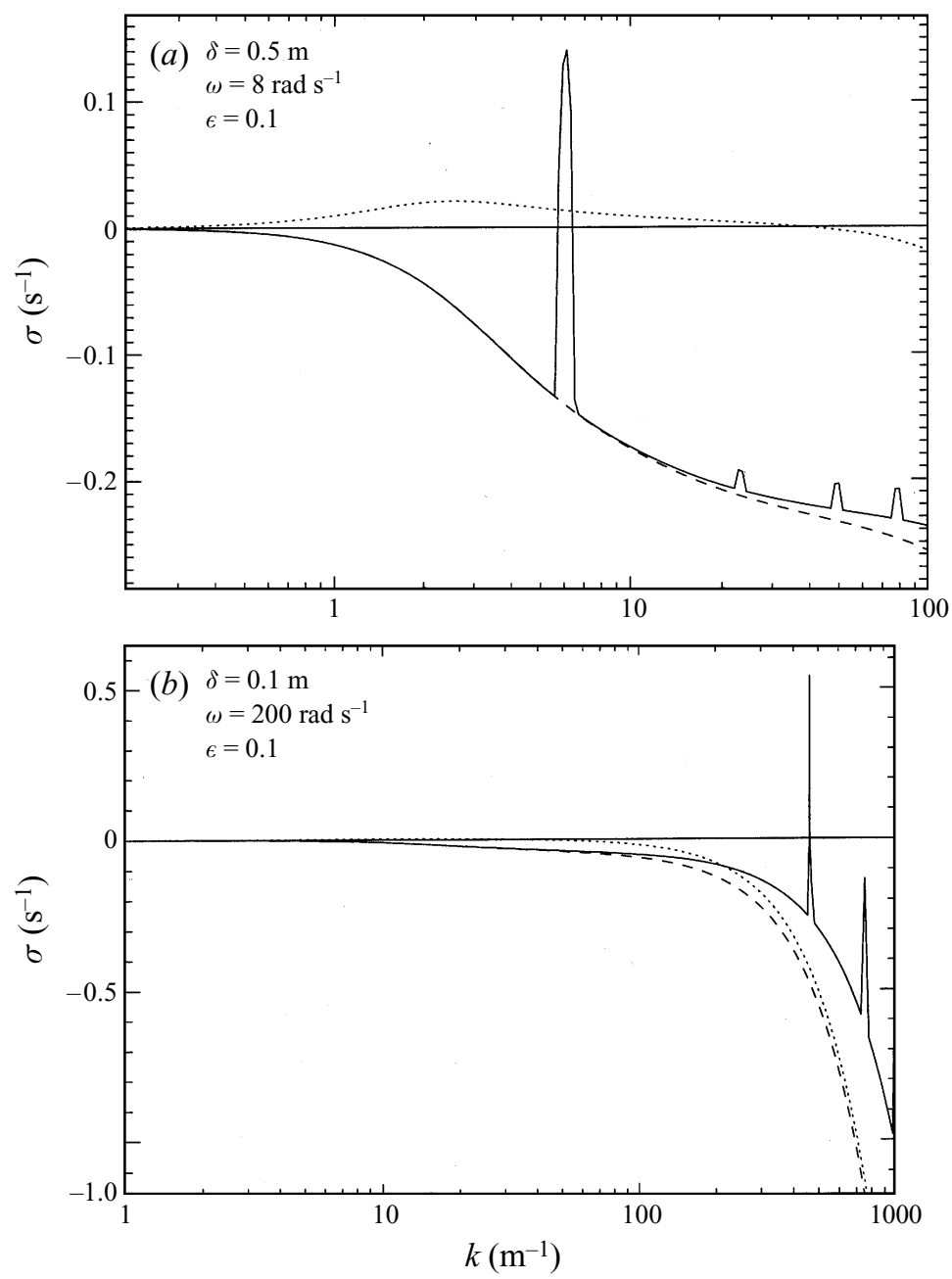

FIGURE 5. Graphs of growth rate $\sigma$ versus wavenumber $k$ when viscous and ohmic damping are included. The solid curve represents the exact growth rate, and the other two curves the approximation (4.10). The dotted curve takes account of viscous damping only, and the dashed curve viscous and ohmic damping.

In figure 5 we show some typical results taking $\rho=2 \times 10^{3}, \lambda=1, \gamma=0.7$, and $v=10^{-6}$ in SI units. The approximation (4.10) predicts that ohmic damping has the dramatic effect of completely stabilizing the system (cf. McHale \& Melcher 1982). As expected however this approximation fails in the vicinity of the resonance points, where the exact method predicts an unstable band of wavelengths. This band becomes narrower as the field strength decreases, or as $\omega$ increases, but the maximum growth rate increases with $\omega$.

To gain further insight, we plot in figure 6 both real and imaginary parts of $s$ versus $k$ when there is no damping (cf. figure 4). Generally the oscillatory frequency is much greater than the growth rate, which partly explains the strong damping. It can be seen that close to the resonance point the mode of maximum growth changes, and the oscillation frequency is much smaller. 


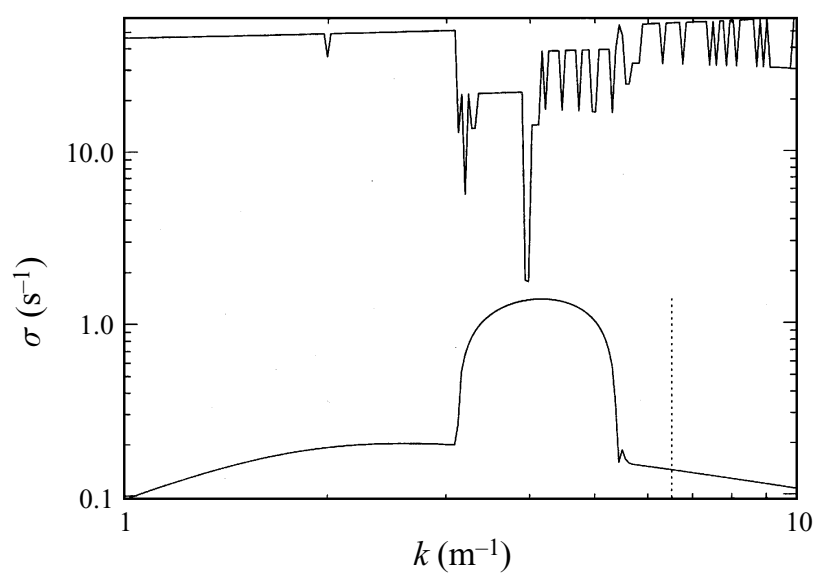

FIGURE 6. Graphs of $\operatorname{Im}(s)$ (lower curve) and the corresponding $\operatorname{Re}(s)$ (upper curve) versus wavenumber $k$, for the mode with maximum growth rate. $\delta=0.5 \mathrm{~m}, \omega=8 \mathrm{rad} \mathrm{s}^{-1}, \epsilon=1.0$.

Behaviour near the resonance points can be estimated using the method of $\S 2.3$, and the new form of (3.5) is

$$
\delta_{s}=\delta_{w}+\frac{1}{2} \mathrm{i}\left(S / \epsilon-\hat{a}_{0 I}+C_{D} \hat{d}_{0 R}\right) \pm \frac{1}{2}\left[\left(2 \delta_{w}-\hat{a}_{0 R}-C_{D} \hat{d}_{0 I}\right)^{2}-\left|\hat{a}_{-1}\right|^{2}\right]^{1 / 2},
$$

where $S=2 v k^{2} / \Omega$ is the dimensionless parameter of viscous damping. As before the maximum growth rate occurs slightly away from the resonance point, where

$$
\delta_{w}=\frac{1}{2}\left(\hat{a}_{0 R}+C_{D} \hat{d}_{0 R}\right)
$$

and the maximum growth rate is

$$
\epsilon \delta_{s}=\frac{1}{2} \epsilon\left[\left|\hat{a}_{-1}\right|+\hat{a}_{0 I}-C_{D} \hat{d}_{0 R}\right]-\frac{1}{2} S .
$$

This expression is linear in $\epsilon$, and is negative when $\epsilon=0$ because of viscous damping. There is therefore a minimum or critical $\epsilon=\epsilon_{c}$ say, necessary to induce instability, given by

$$
\epsilon_{c}=S /\left(\left|\hat{a}_{-1}\right|+\hat{a}_{0 I}-C_{D} \hat{d}_{0 R}\right)
$$

The corresponding critical value of $B_{0}, B_{c}$ say $=\left(2 \epsilon_{c} \mu_{0} \rho g \delta / \Gamma\right)^{1 / 2}$. A graph of $B_{c}$ versus $\omega$ is shown in figure 7. Figure 8 plots maximum growth rate against $\omega$ for various values of $M$. Note that the maximum growth peaks move slightly to the right as $\epsilon$ decreases; this a consequence of the resonant frequency shift described by (3.6).

\section{Conclusions}

Previous theoretical work on the instability of a liquid-metal free surface induced by a parallel alternating magnetic field has been extended in two main directions. Relaxing the usual high-frequency assumption has highlighted the importance of resonance; generally growth rates are small, but increase dramatically near the resonance points where $\Omega=m \omega$. Abandoning also the approximation $R_{m}=0$ has shown that ohmic damping is much more effective than viscous dissipation in limiting wave growth. Indeed all modes are suppressed except for a narrow band of wavenumbers in the vicinity of the first resonance point (at least in the case of relatively weak fields). 


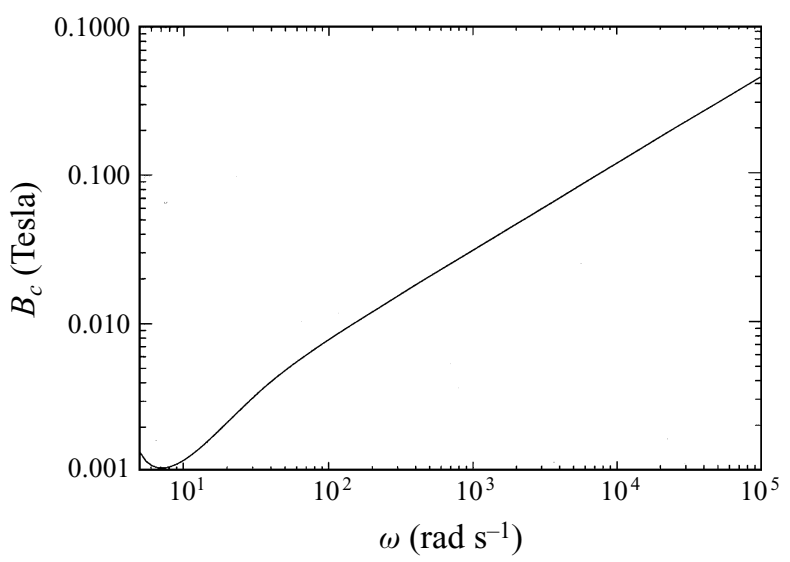

FIGURE 7. Graph of critical magnetic field strength $B_{c}$ versus $\omega$.

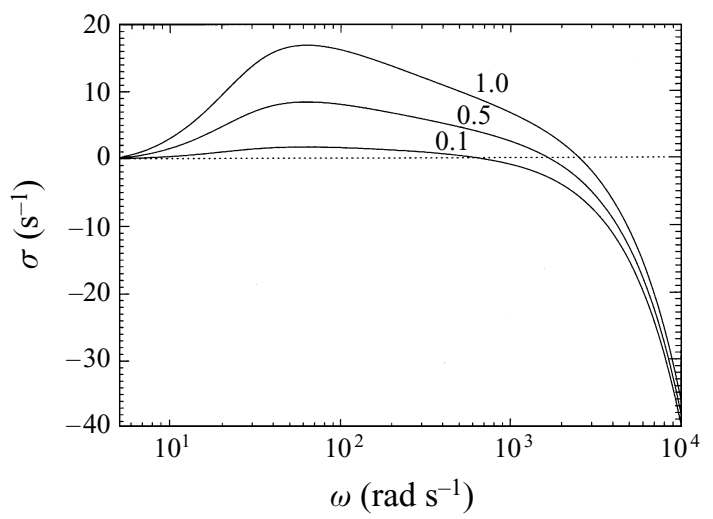

FIGURE 8. Graphs of maximum growth rate versus $\omega$. The number beside each curve is the value of the magnetic field strength parameter $M$.

The weak method of formulation leads to a single generalized Mathieu-Hill equation describing the evolution of the free surface. This provides relatively simple methods of estimating growth rates, which can in most cases be approximated by algebraic formulae.

It would be interesting to compare our theory with experiment, but the data presently available relate to somewhat different systems. Hull, Wiencek \& Roe (1989) have studied experimentally electromagnetic levitation of a liquid metal layer, and the Rayleigh-Taylor instability. Our results are at least consistent with their finding that the metal layer is most unstable when the wavenumber vector is perpendicular to the applied field. In our analysis this would correspond to setting $\theta=\frac{1}{2} \pi$, giving $M=0$ so that waves are purely hydrodynamic (cf. (2.25)).

This paper was partly conceived when one of the authors (Y.F.) was visiting the Mathematics Department of the University of Waikato. The support and hospitality of this university is gratefully acknowledged. 


\section{REFERENCES}

DeEPaK \& Evans, J. W. 1995 The stability of an interface between viscous fluids subjected to a high-frequency magnetic field and consequences for electromagnetic casting. J. Fluid Mech. 287, 133-150.

FAUTRELle, Y. 1991 Free surface electromagnetic instabilities in liquid metals. In Magnetohydrodynamics in Process Metallurgy (ed. J. Szekely, J. W. Evans, K. Blazek \& N. El-Kaddah), pp. 63-68. The Minerals, Metals \& Materials Society.

GARNiER, M. \& MOREAU, R. 1983 Effect of finite conductivity on the inviscid stability of interface submitted to a high frequency magnetic field. J. Fluid Mech. 127, 365-377.

Hull, J., Wiencek, T. \& Roe, D. M. 1989 Magnetohydrodynamic stability in the electromagnetic levitation of horizontal molten-metal sheets. Phys. Fluids 1, 1069-1076.

Jordan, D. W. \& Smith, P. 1987 Nonlinear Ordinary Differential Equations. Oxford.

Lamb, H. 1932 Hydrodynamics. Cambridge University Press.

McHale, E. J. \& Melcher, J. R. 1982 Instability of a planar liquid layer in an alternating magnetic field. J. Fluid Mech. 114, 27-40.

Ramos, A. \& Castellanos, A. 1996 Effect of viscosity on the stability of an interface subjected to high-frequency magnetic fields. Phys. Fluids 8, 1907-1916.

SAGARDIA, S. R. 1974 Electromagnetic levitation melting of large conductive loads. PhD thesis, University of Toronto.

SNEYD, A. D. 1993 Theory of electromagnetic stirring by AC fields. IMA J. Maths Appl. Business Industry 5, 87-113.

Sneyd, A. D. 1996 Systems of Mathieu-Hill type equations. University of Waikato Mathematics Research Rep. 48, Series II.

Titchmarsh, E. C. 1939 The Theory of Functions. Oxford. 